\title{
Two-dimensional chiral model for liquid crystals, bent hard needles: A Monte Carlo simulation
}

\author{
Jorge Peón, Jimena Saucedo-Zugazagoitia, \\ Fabian Pucheta-Mendez, and Raúl A. Perusquía \\ Instituto de Química, Universidad Nacional Autónoma de México, México 04510 Distrito Federal, México \\ Godehard Sutmann \\ Central Institute for Applied Mathematics (ZAM) and John von Neumann Institute for Computing (NIC), \\ Research Centre, Jülich (FZJ), D-52425, Jülich, Germany \\ Jacqueline Quintana- $\mathrm{H}^{\mathrm{a}}$ \\ Instituto de Química, Universidad Nacional Autónoma, México, México 04510 Distrito Federal, México
}

(Received 19 October 2005; accepted 27 July 2006; published online 14 September 2006)

\begin{abstract}
The liquid crystalline behavior of a two dimensional (2D) model of hard needles bent into a "zigzag shape" is studied. This model, originally designed to study two dimensional chiral segregation, also shows liquid crystalline behavior and has some anomalous features which are contrasted in relation to the following: (i) Most of the microscopical models used to study liquid crystals have a symmetry axis that coincides with a molecular axis; (ii) in three-dimensions, chiral molecules can form cholesteric instead of nematic phases; (iii) the smectic phase is usually found when attractions are present or at least when the molecules have finite volume. Despite the fact that the present 2D model does not have any of these characteristics, numerical evidence is found for the occurrence of nematic and smectic phases. Since these molecules are athermal, infinitely repulsive, and infinitesimally thin, the liquid crystalline characteristics are attributed to excluded volume effects. To determine the mesophases of the model, both nematic and smectic order parameters as well as distribution functions are computed. (C) 2006 American Institute of Physics.
\end{abstract}

[DOI: $10.1063 / 1.2338313$ ]

\section{INTRODUCTION}

It is well known that dimensionality greatly affects the nature of the phase diagram of statistical-mechanical systems. Before results are discussed some differences between two and three dimensional liquid crystalline phase behavior are mentioned. In this respect the two-dimensional (2D) system studied here, the bent hard needles model, ${ }^{1}$ is helpful for illustrating some of these differences. In the first place, some characteristics of the model are mentioned which will disappear if the model is brought to three dimensions (3Ds), and which consequently would change some phase properties. Some of the differences are discussed regarding phase properties between the present model and those of other comparable models.

The model studied here was originally designed for the investigation of $2 \mathrm{D}$ chiral segregation ${ }^{1}$ and shows the feature that when considered in three dimensional space, i.e., three translational and rotational degrees of freedom, the chiral features disappear. On the other hand, in 3D the molecules can generate solids of revolution with a symmetry axis, a situation that would be impossible when embedded in $2 \mathrm{D}$ space. In the limiting case when the so called arm length tends to zero, the bent hard needles transform into the hard needles of Frenkel and Eppenga, ${ }^{2}$ the nematic phase, stable

\footnotetext{
${ }^{a)}$ Author to whom correspondence should be addressed. Electronic mail: jaq@correo.unam.mx
}

in three dimensions, develops only quasi-long ranged order. In 3D, chiral molecules can generate cholesteric phases while nonchiral ones produce nematics. Since in this paper a 2D model is considered, the chirality cannot induce cholesteric phases, but instead the system can generate nematics. Also, the phase behavior of this model displays important differences when compared to those of other well studied models. For example, it allows for the occurrence of smectic phases which are not present in the 2D model of hard needless studied by Frenkel and Eppenga. ${ }^{2}$

In the literature, several studies of liquid crystalline mesophases can be found occurring in 3D models. With regards to anisotropic continuous potentials, these models can be classified into several categories, e.g., those which take into account attractive and/or repulsive interactions between molecules, including van der Waals and electrostatic interactions, ${ }^{3}$ which produce a nontrivial temperature dependence of properties in the system. Although complicated, the usefulness of this kind of potentials is their ability to model many different situations. Some examples are the Kihara fluid model studied recently ${ }^{4}$ and the Gay-Berne potential which has been widely studied and applied to different problems, as for example, adsorption near a graphite surface, ${ }^{5}$ chiral induction, ${ }^{6}$ ferroelectric nematics, ${ }^{7}$ or discotics, quadrupolar, or biaxial liquid crystals. ${ }^{8}$ The Gay-Berne potential has also been modified in order to study the effect of the shape of the molecules on the type of smectic phases.

Likewise, the liquid-crystalline phases displayed by less 


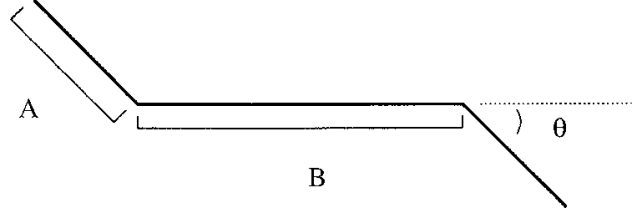

FIG. 1. Scheme of the bent hard needle model. The length of the molecule is defined as $2 A+B=1$, where $A$ is called the arm and $B$ the body. The orientation of the arm with respect to the body is denoted by $\theta$.

complex models have also been determined. This is the case of models where interactions consider only infinite repulsions, such as hard ellipsoids and spherocylinders (see, e.g., the properties reported for these two models by Frenkel et $\left.a l^{9,10}\right)$. Within this group, the simplest $2 \mathrm{D}$ model is presumably the hard needles model, ${ }^{2}$ which has infinitely repulsive interactions and molecules are infinitely thin, i.e., they have no effective volume. Because of their extreme simplicity, these models are useful to understand the relationship between the occurrence of equilibrium phases and very basic molecular features. The model studied here can be classified within this category.

In 1949 Onsager ${ }^{11}$ applied a mean-field-type theory to study the isotropic to nematic symmetry breaking for hard rods in $3 \mathrm{D}$ in the limit of divergent length to thickness ratio. According to that formalism, Onsager has shown that the existence of a long-ranged nematic phase is induced only by excluded volume effects. In general, statistical-mechanical studies for 3D liquid crystals have in common the relevant role of the repulsive part of the intermolecular potential in generating the orientational order present in the nematic phase. However, this conclusion cannot be generalized in a straightforward manner to the equivalent two dimensional system. In particular, a theory of the Onsager type for needles in $2 \mathrm{D}$ does not lead to accurate predictions for the isotropic-nematic transition. Furthermore, the work of Frenkel and Eppenga ${ }^{2}$ provides important arguments that indicate the absence of genuine long ranged order for hard needles in 2D.

In 1988 Frenkel et al. ${ }^{10}$ confirmed the existence of smectic phases for 3D hard spherocylinders, so that, and contrary to what was believed then, attractive interactions seem not to be a necessary ingredient to stabilize these structures. Later on, along the same lines, Vanakaras and Photinos ${ }^{12}$ studied a hard core 3D model with molecules composed of three different segments that also gave rise to smectic phases. In contrast, for the model presented here, we mention that even though the volume of a molecule is zero, the smectic phase develops because of the existence of an excluded volume.

As expected, the appearance of different liquid crystalline phases in the present model depends on the packing density and on the specific molecular shape, given by the molecular length of the $\operatorname{arm} A$ and angle $\theta$ (see Fig. 1 and text below). Due to these geometric parameters, a more rich map of mesophases is found compared to those obtained by Frenkel and Eppenga ${ }^{2}$ for hard needles. Despite the simplicity of the model, it should be stressed out that a relatively complex phase map, with nematic and smectic phases is produced.
The main goal of this work is to find the importance of the particular features of the bent hard needles model for the occurrence of liquid crystalline mesophases. Constant volume and constant pressure Monte Carlo simulations were performed in order to draw a map for the mesophases of the bent hard needles model. To determine the appearance of the different phases both nematic $S$ and smectic $\Lambda_{\text {sm }}$ order parameters are calculated as well as characteristic distribution functions to complement the conclusions.

The paper is organized as follows: The model and the details of simulations are contained in Sec. II, results are presented in Sec. III, and finally Sec. IV is dedicated to conclusions.

\section{HARD BENT NEEDLES MODEL AND SIMULATIONS DETAILS}

The two-dimensional model considered here was originally designed to study chiral segregation. It may be considered as a generalization of the hard-needle model initially studied by Onsager $^{11}$ and later on by Frenkel and Eppenga. ${ }^{2}$ More specifically, a molecule is constructed by bending each side of a hard needle by an angle $\theta$ and of length $A$. The parameters $A$ and $\theta$ thereby completely define the model, since the length of the body $B$ of the molecule is taken such that the total length of the molecule is $1=2 A+B$. A schematic of the molecule, explaining its relevant parameters is shown in Fig. 1.

The molecules are infinitely thin and possess infinitely hard bodies, so that interactions are due to their excluded volume, depending on the molecular parameters $A, B$, and $\theta$. The intermolecular potential energy $U_{i j}$ is given by

$$
U_{i j}= \begin{cases}\infty, & \text { overlap } \\ 0, & \text { no overlap. }\end{cases}
$$

Under certain thermodynamic conditions the geometry of the molecules allows the development of liquid crystalline features.

Constant volume and constant pressure Monte Carlo simulations using the Metropolis acceptance criterion, in the present case, reduce to the rule of accepting all nonoverlapping configurations. For all simulations standard twodimensional periodic boundary conditions were applied and the molecular angle was fixed to $\theta=90^{\circ}$. Taking into account that $2 A+B=1$, the range of values allowed for the molecular arm length is $A \in[0,0.5]$. ${ }^{1}$ Simulations were carried out at different densities, defined as $\rho=N / L^{2}$, where $L$ is the simulation box length.

For constant volume simulations the acceptance criterion is reduced to a simple rule, i.e., a configuration is accepted if there are no overlaps between pairs of molecules, since molecules exhibit an infinite hard core. Each trial configuration is realized by choosing at random a molecule and randomly deciding if it is translated or rotated. The maximum displacement in the center-of-mass coordinates and rotation angle was established by adjusting the acceptance ratio along the simulation to approximately $30 \%$ of the trial moves. For constant volume simulations the box was a perfect square of side $L$. Several values of the arm length $A$ were chosen and for 
TABLE I. Parameters used for simulations; for each molecular arm $A$ $\in[0,0.5]$ we selected a series of densities; the number of particles was $N$ $=1000$.

\begin{tabular}{lc}
\hline \hline Molecular arm $A$ & Density $\rho$ \\
\hline 0.0625 & $5,7,9,11,13,15,17,20,25,30,35,40$ \\
0.11 & $9,10,11,12,15,20,25,30,40$ \\
0.115 & $9,11,12,15,20,25$ \\
0.12 & $9,10,11,12,15,20,35$ \\
0.1253 & $9,10,11,12,15,20,25$ \\
0.18 & $5,7,9,11,15,17,20,25,30,35$ \\
0.25 & $1,2,5,7,10,13,14,15,17,20,25$ \\
0.3334 & $5,7,9,11,13,15,20,25,30$ \\
0.4 & $5,7,9,11,15,17,20,25,35,40$ \\
0.46 & $5,7,9,11,15,17,20,25,35$ \\
\hline \hline
\end{tabular}

each case different densities were chosen in order to explore the phase map, thereby identifying regions of isotropic, nematic, and smectic phases. Table I includes the parameters considered for constant volume simulations. For most of the simulations the number of particles was $N=1000$. In order to study finite size effects the number of particles was varied for a particular arm length $A=0.3334$.

For the particular case of $A=0.18$, constant pressure simulations were also performed using a rectangular simulation box. In this case, a trial configuration was realized by choosing at random a particle and randomly deciding if a change in the volume or a particle movement is tested. For a particle move, either a translation or a rotation was chosen with an equal probability.

In order to test the quality of equilibration, most of the runs were started from two different initial configurations, one of them completely disordered as in the liquid phase, while the other one was started with orientational order as in a nematic phase. Equilibrium was assumed when properties of both simulations became stable and coincided with each other. The parameters used to check the criterion for equilibrium were the nematic, $S$, and smectic, $\Lambda_{\mathrm{sm}}$, order parameters. Although expensive, this route increases the certainty about reaching the equilibrium state. Several millions of configurations were generated for both equilibration and production.

In two dimensions the nematic order parameter $S$ can be computed as follows: ${ }^{2}$

$$
S=\frac{1}{N}\left\langle\sum_{i=1}^{N} \cos \left(2 \vartheta_{i}\right)\right\rangle
$$

where $\vartheta_{i}$ is the angle between the body $B$ of the molecule $i$ and the nematic director $\mathbf{n}$, and the angular brackets denote the configurational average, where $\mathbf{n}$ can be obtained according to Ref. 2.

The smectic order parameter is defined as the Fourier transform of the density of particles ${ }^{13}$

$$
\Delta_{\mathrm{sm}}=\frac{1}{N}\left\langle\left|\sum_{j=1}^{N} e^{i k d_{j}}\right|\right\rangle,
$$

where $N$ is the number of particles, $k=2 \pi / \lambda_{\mathrm{sm}}, \lambda_{\mathrm{sm}}$ is the distance between layers of molecules, and $d_{j}$ is the projection of the coordinates of particle $i$ onto the direction of the smectic director $\mathbf{s}$, which is actually orthogonal to the layers. In Appendix A, an efficient method is proposed to calculate nematic and smectic order parameters.

To analyze in more detail the structure of different phases, the parallel $g_{\|}$and perpendicular $g_{\perp}$ distribution functions are calculated, which are defined as

$$
g_{\otimes}(\mathbf{r})=\frac{1}{N \rho}\left\langle\sum_{i}^{N} \sum_{j \neq i}^{N} \delta\left(\mathcal{P}_{\otimes}\left(\mathbf{r}+\mathbf{r}_{j}-\mathbf{r}_{i}\right)\right\rangle,\right.
$$

where $\delta$ is the Dirac $\delta$ function, $\otimes=\{\|, \perp\}, \mathcal{P}_{\|}(\mathbf{x})=(\mathbf{x} \cdot \mathbf{s}) \mathbf{s}$ is the projector of position coordinates of the centers of mass of molecules onto the smectic director $\mathbf{s}$, and $\mathcal{P}_{\perp}(\mathbf{x})=(\mathbf{x} \cdot \mathbf{t}) \mathbf{t}$ is the projector perpendicular to $\mathbf{s}$, so that the relation $\mathbf{s} \cdot \mathbf{t}=0$ holds. We note that for the different liquid crystal phases, a characteristic behavior is expected for the distribution functions: (i) isotropic phase: both $g_{\|}$and $g_{\perp}$ are structureless; (ii) nematic phase: also both functions are structureless, since the functions do not contain any information about orientations; (iii) smectic phase: $g_{\|}$shows a periodic pattern, according to the smectic periodicity, while $g_{\perp}$ is structureless. In order to complete all definitions of properties used in this paper, we anticipate some of our results discussed in the next section: (iv) An intermediate phase exists, where smectic layers start to form in separated clusters, which have no common director. In this case, both $g_{\|}$and $g_{\perp}$ show a periodic pattern with decaying amplitude, which indicates short ranged correlations across the clusters. In the transition zone from this region to smectic phase, $g_{\perp}$ starts to lose structural information. In this so called defective smectic region, at intermediate density $\rho$ and arm length $A$, there are groups or piles of molecules that are stacked in such a way that their centers of mass draw a curved line. In these groups, the centers of adjacent particles are so close to each other that as a consequence, the orientation of adjacent molecules is highly correlated. As an attempt to characterize this region and to provide a quantitative way to differentiate it from the isotropic one, the fraction number $\Phi_{\mathrm{fr}}$ was defined as the average number of molecules which have at least one close neighbor relative to the total number of molecules $N{ }^{14}$ Thereby a molecule $j$ is defined as a close neighbor of molecule $i$ if its center lies inside of a circle with radius $R$ centered at $i$. The distance $R$ is defined through molecular parameters as the minimum between the arm length $A$ and half of the body $B$ of the molecule and $R \equiv \min (A, B / 2)$, i.e.,

$$
\Phi_{\mathrm{fr}}=\frac{1}{N}\left\langle\sum_{i} 1 ; \exists j:\left|\mathbf{r}_{i}-\mathbf{r}_{j}\right| \leqslant R\right\rangle .
$$

Because of its definition it is a short range order property and not a real order parameter. However, it gives a manifestation of the formation of the cumulus of molecules where their centers are sufficiently close. In a very dense phase with layers of molecules, $\Phi_{\mathrm{fr}} \rightarrow 1$ because most molecules would have at least one neighbor which is very close, while on the other hand, in a very dilute phase $\Phi_{\mathrm{fr}} \rightarrow 0$ (in Appendix B the maximum orientation between two molecules is calculated, obeying this criterion). 


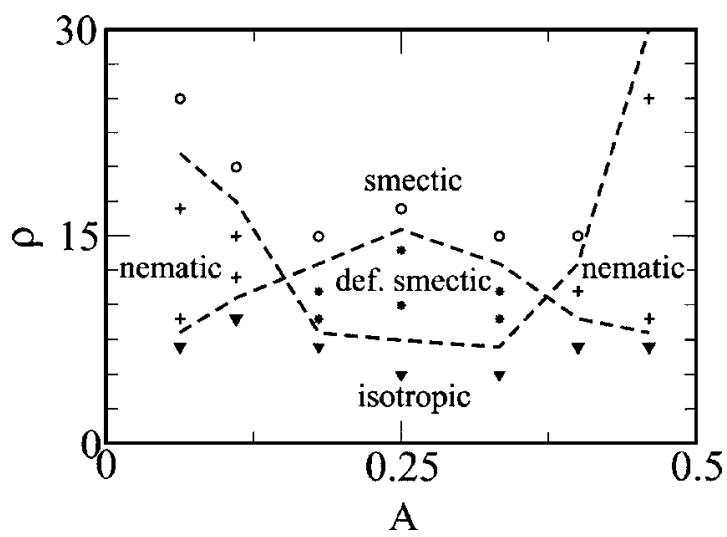

FIG. 2. Map of mesophases of the bent hard needle model: isotropic (triangles), nematic (crosses), defective smectic (stars), and smectic (circles). Different regions are shown as function of number density $\rho$ vs arm length $A$. Phases were identified according to characteristic parameters, i.e., nematic order parameter, smectic order parameter, $\Phi_{\mathrm{fr}}$ function, and both parallel $g_{\|}$and perpendicular $g_{\perp}$ distribution functions.

\section{RESULTS}

Most of the results were obtained using constant volume Monte Carlo simulations. For the particular arm length $A$ $=0.18$, constant pressure Monte Carlo was used additionally as a check, as discussed in Sec. III F. The molecular angle in all cases was fixed to $\theta=90^{\circ}$. Figure 2 shows a map of the different phases obtained with constant volume Monte Carlo, where the density, $\rho$, is plotted versus molecular arm length $A$. For each value of $A$ simulations were performed for a series of densities starting from low $(\rho \approx 5)$ up to high values $(\rho \approx 40)$. For the series of densities studied, only those points are shown which are closest to the border lines between different regions. The dotted lines are plotted as a guide to the eye in order to indicate the transition zone between different regions. Note that these lines are not to be understood as phase coexistence lines, but are drawn as midpoints between distinct phase points. As can be seen from Fig. 2, for sufficiently low density an isotropic phase is found for all values of $A$. Triangles correspond to the highest density found for this phase. For intermediate densities, the different regions depend strongly on the value of $A$. The nematic phase that is shown by crosses in the phase map is located in those regions where the values of $A$ are either close to zero or close to $1 / 2$, i.e., very small or very large values of the arm length. In both cases molecules have a needlelike shape $(A / B \gg 1$ or $A / B \ll 1)$ and therefore they have similar properties to the hard needles of Frenkel and Eppenga, ${ }^{2}$ i.e., they show a nematic behavior. On the other hand, when the shape of the molecules is not needlelike, $A / B \approx 1$, they do not have nematic behavior and instead the molecules aggregate in curved layers, what is called here a defective smectic region. For example, for values of arm length around $A=0.25$ (in the center of the figure), no nematic phase is found but instead there are smecticlike domains, where the layers of molecules follow a curved path (defective smectic region). This region is located in between the isotropic and the smectic region. Stars indicate the points for which this region was found. More explicitly, for intermediate values of arm length $A$ the defective smectic region appears instead of the nematic phase. Both (a)
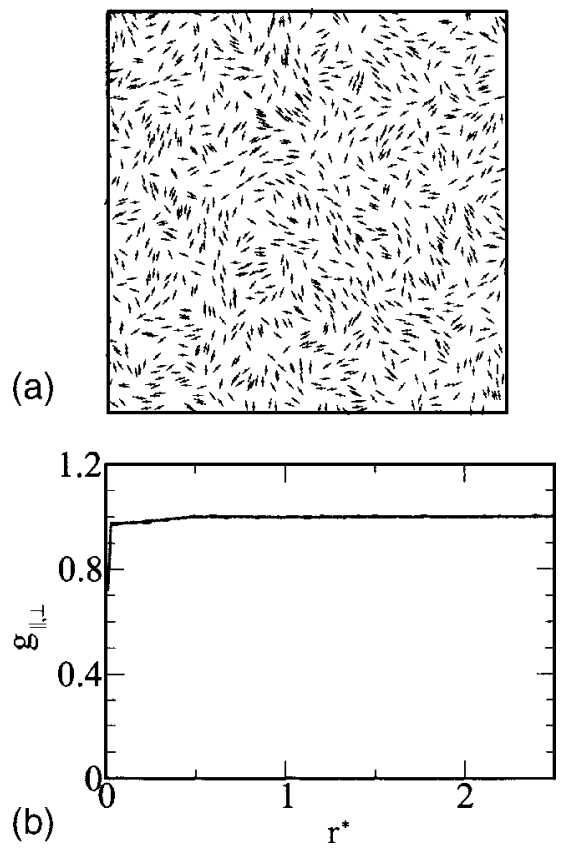

FIG. 3. Properties for a typical case of the isotropic phase corresponding to molecular arm $A=0.0625$ and density $\rho=5$. (a) Snaphot of one of the final configurations, and (b) the parallel $g_{\|}$(dotted line) and perpendicular $g_{\perp}$ (solid line) distribution functions.

the defective smectic region and the nematic phase are located between the isotropic and smectic phases. Finally, if the density is sufficiently high the smectic behavior is found, for all values of the molecular arm length $A$, except the limiting cases $A=0$ and $A=0.5$. Circles are plotted for the states of smallest density corresponding to the smectic phase. As shown below, a manifestation of this behavior can be found for the smallest value of arm length studied $(A=0.0625)$ where a significant indication of the appearance of smectic

(a)
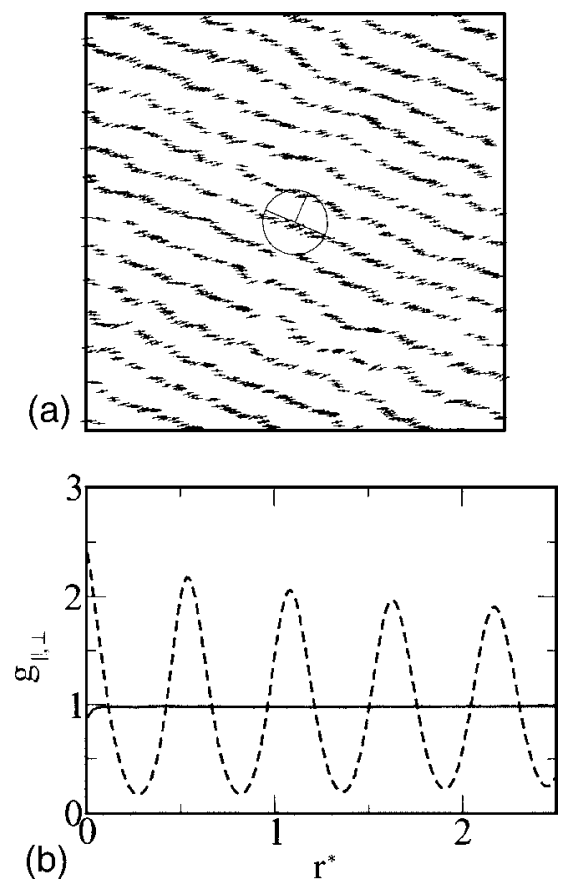

FIG. 4. Same set of properties as in Fig. 3 for the smectic phase corresponding to molecular arm $A=0.25$ and density $\rho=20$. 
(a)
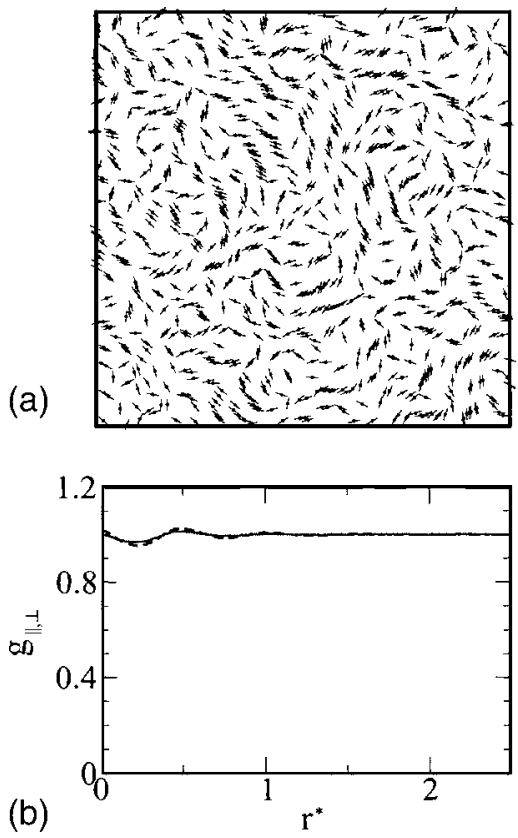

(c)

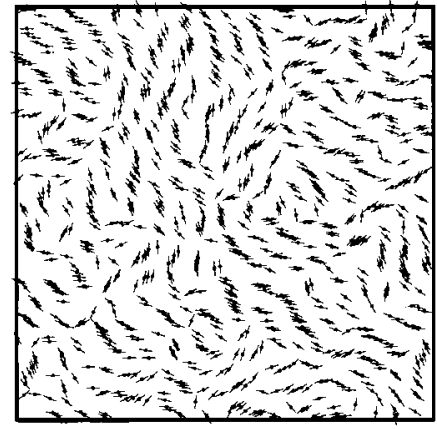

(d)

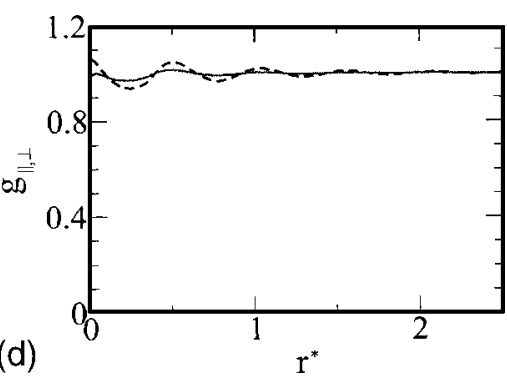

FIG. 5. Same set of properties as in Fig. 3 for the defective smectic phase corresponding to molecular arm $A=0.3334$ and density $\rho=9$ (a) and (b) and for $\rho=11$ (c) and (d). phase is found for sufficiently high density, namely, $\rho=40$. All conclusions mentioned above are based on the evidence from the corresponding snapshots, nematic and smectic order parameters $S$ and $\Lambda_{\mathrm{sm}}$, respectively, as well as the parallel and perpendicular distribution functions, $g_{\|}(r)$ and $g_{\perp}(r)$, shown in Figs. 3-6 discussed below.

\section{A. Isotropic phase}

Figure 3 shows several properties for a typical case from the isotropic region, $\rho=5$ and $A=0.0625 .^{15}$ In Fig. 3(a) one of the final configurations is shown from simulations where for clarity only a fraction of the body $B$ of the molecule is displayed and the center of mass is represented by a solid circle. ${ }^{16}$ Figure $3(\mathrm{~b})$ shows both the parallel $g_{\|}$and perpendicular $g_{\perp}$ distribution functions. ${ }^{17}$ Both of them exhibit practically the same features: they reveal a random, structureless behavior which indicates an isotropic phase. Structural properties and order parameters are shown in Fig. 7(a). The nematic order parameter $S$, smectic order parameter $\Lambda_{\text {sm }}$, and the fraction $\Phi_{\mathrm{fr}}$ are plotted as a function of density. As is seen for $\rho=5$ the values of the parameters are quite small, characteristic of an isotropic phase. Also the fraction function $\Phi_{\text {fr }}$ is very small, reflecting the fact that in the low density phase it is unlikely to build up clusters with short range correlations.

\section{B. Smectic phase}

As representative for the smectic region the arm length $A=0.25$ and density $\rho=20$ is chosen (see Fig. 4). In Fig. 4(a) a snapshot of one of the final configurations is shown. In Fig. 4(b) the parallel and perpendicular distribution functions, $g_{\|}$ and $g_{\perp}$, are displayed. As is clear from the figure, a strong difference exists between the distribution functions. While $g_{\perp}$ exhibits no structure, showing an isotropic distribution of molecules perpendicular to the smectic director, $g_{\|}$exhibits decaying oscillations with periodicity, $\lambda_{\mathrm{sm}}$. In an ideally developed smectic phase, oscillations would not decrease at all due to a stable periodic pattern. Therefore, decaying oscillations with a decay length $\xi>1$ are indicative for an emerging smectic phase (from a fitting procedure a value of $\xi \approx 5$ was obtained). The decay length $\xi$ is defined here as the exponential decay of the upper envelope, $f(r)$, of the oscillations, i.e.,

$$
f(r)=1+c e^{-r / \xi},
$$

where $c$ is the amplitude at $r=0$. In Fig. 7(b) the nematic $S$ and smectic $\Lambda_{\mathrm{sm}}$ order parameters are displayed as well as the fraction function $\Phi_{\mathrm{fr}}$ as a function of density $\rho$. As the density $\rho$ increases, the nematic order parameter $S$ tends to large values $S \approx 1 .{ }^{18}$ It can be seen that the smectic order

(a)
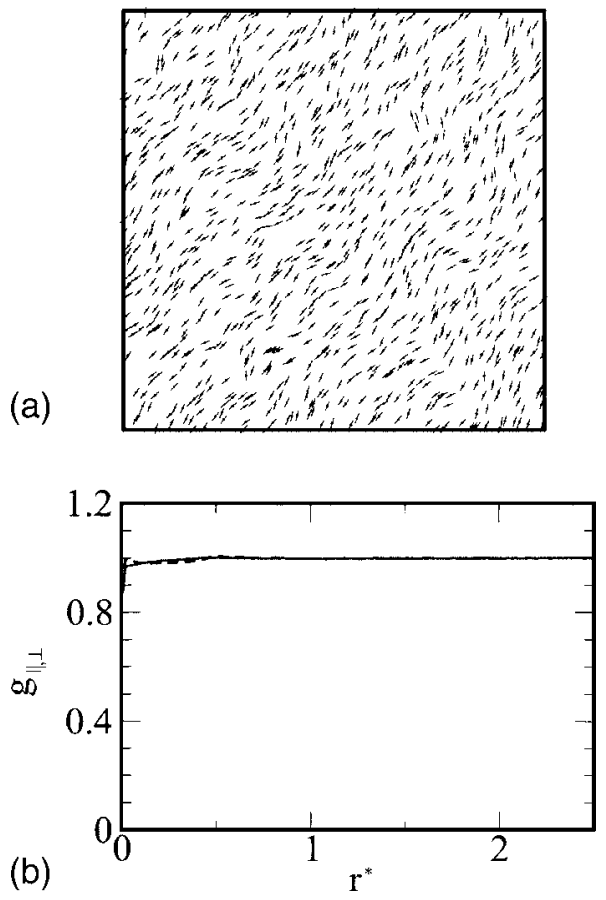

FIG. 6. Same set of properties as in Fig. 3 for the nematic phase corresponding to molecular arm $A=0.46$ and density $\rho=11$. 


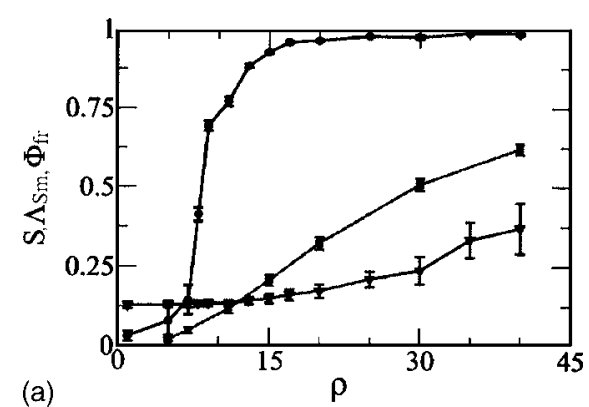

(a)

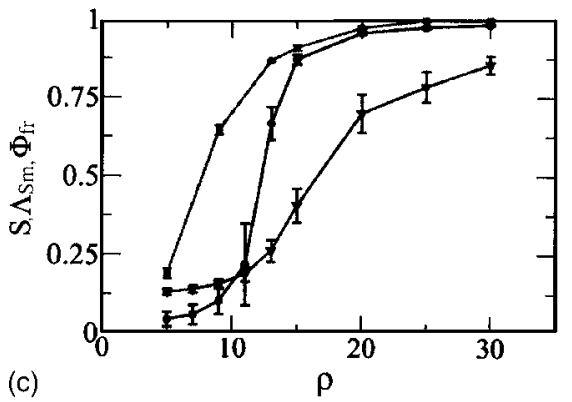

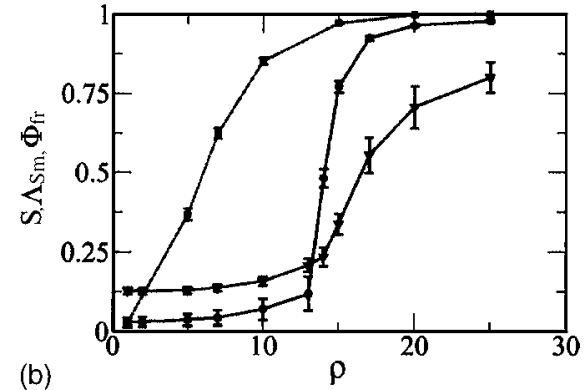

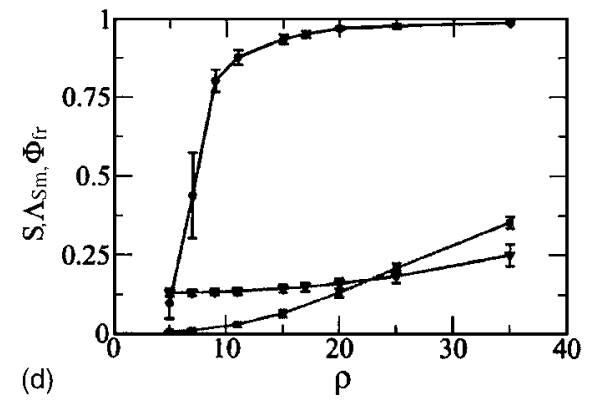

FIG. 7. Density dependence of the nematic (solid circles) and smectic (solid triangles) order parameters $S$, $\Lambda_{\text {sm }}$, and fraction $\Phi_{\text {fr }}$ (solid squares) for molecular arm, (a) $A=0.0625$, (b) $A=0.25$, (c) $A=0.3334$, and (d) $A$ $=0.46$. parameter, $\Lambda_{\mathrm{sm}}$, increases as well but at the largest density studied, $\rho=25$, it has not yet saturated. This indicates a strong orientational order and significant periodic positional ordering in one of the directions of the simulation box, actually in the direction of the smectic director. In contrast to the isotropic case, the fraction function $\Phi_{\mathrm{fr}}$ is rising faster than both other order parameters and it seems to be saturated for $\rho \approx 15$. This means that for this arm length, $A=0.25$, local structures, characterized by close neighbors, appear already for small densities, leading to a formation of small clusters, which have no common orientation for this regime of low density. This is the reason why $S$ and $\Lambda_{\text {sm }}$ get larger values only at larger densities. The fact that $\Phi_{\text {fr }}$ raises fast means that, as the density increases, the molecules start to assemble among themselves by generating layers, thereby preventing displacements along the body of molecules and limiting the formation of nematic structure (see below). This behavior was found to be characteristic for arm lengths in the center of the phase map, i.e., in the vicinity of $A \approx 0.25$.

\section{Defective smectic region}

Figure 5 corresponds to the case of the defective smectic region where the layers of molecules are curved. To show the characteristics of this region, densities $\rho=9$ and $\rho=11$ are analyzed for a molecular arm length $A=0.3334$. Figures 5(a) and 5(c) contain snapshots showing that molecules are arranged in layers which follow curved patterns. In the case of the lower density, $\rho=9$, the curvature of the layers is more pronounced than that for the case of density $\rho=11$. Although the difference is not large, it is well established. Figures 5(b) and $5(\mathrm{~d})$ contain information of the distribution functions for $\rho=9$ and $\rho=11$, respectively. The parallel $g_{\|}(r)$ and perpendicular $g_{\perp}(r)$ distribution functions are represented by solid and dotted lines, respectively. It is worth to mention that the direction used to calculate the parallel and perpendicular distribution functions is that defined by the smectic director $\mathbf{s}$. The criterion used to define the direction of $\mathbf{s}$ is based on a maximization procedure. In this region, where curved layers of molecules are formed, the maximization procedure produces a result when the layers of molecules in the system show certain alignment among them. In this region, the transition from isotropic to smectic takes place quite slowly as the density increases. That is, the effect starts from being very small, until the density increases sufficiently that all layers are straight and parallel to each other, corresponding to the smectic phase. More specifically, from the distribution functions for the higher density, $\rho=11$, Fig. 5(d), it is possible to see that a slightly dominant direction emerges that allows to define a smectic director which finally produces that $g_{\|}(r)$ is more structured than $g_{\perp}(r)$. For the lower density $\rho=9$ this is not possible; the smectic director $\mathbf{s}$ is not as clearly defined and therefore there is not an evident difference between the parallel and perpendicular distribution functions. As seen in the Fig. 5(c) both functions show a very similar behavior, i.e., a damped oscillation indicative for short range correlations. For this case the decay length is found to be of the order $\xi \approx 1$. Both functions show a similar behavior which is a sign for an isotropic distribution of small clusters, which is consistent with the findings shown in the corresponding snapshots. That is, in the defective smectic zone the anisotropy develops in a continuous way until the density is sufficiently high. It is to be expected that when the density increases, approaching the smectic region in the phase map, the perpendicular distribution function will lose its features while the parallel one will get more structured. Therefore, the defective smectic region may be considered as a smooth transition zone from the isotropic phase into the smectic one. Figure 7(c) shows the nematic $S$, and the smectic $\Lambda_{\mathrm{sm}}$ order parameters, and the fraction function $\Phi_{\mathrm{fr}}$ as a function of density. It can be seen that $S$ and $\Lambda_{\text {sm }}$ remain relatively small even for densities of about $\rho \approx 12$. Both order parameters have a noticeable increment in approximately the same interval of densities, namely, $\rho \in[13,19]$. On the contrary, the fraction function $\Phi_{\mathrm{fr}}$ increases remarkably fast, actually only for the range of values around $A \approx 0.25$, at the center of the phase map, and as a matter of fact the function 


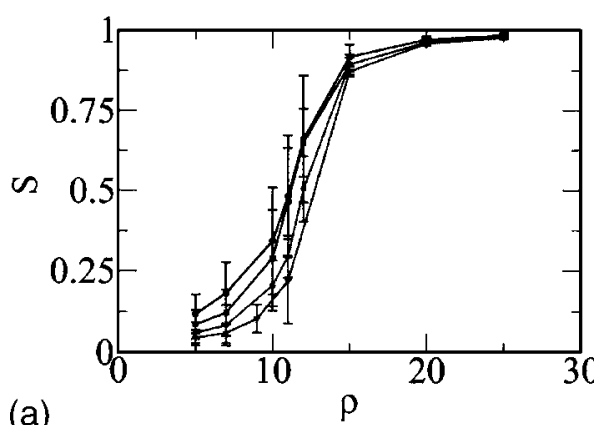

(a)

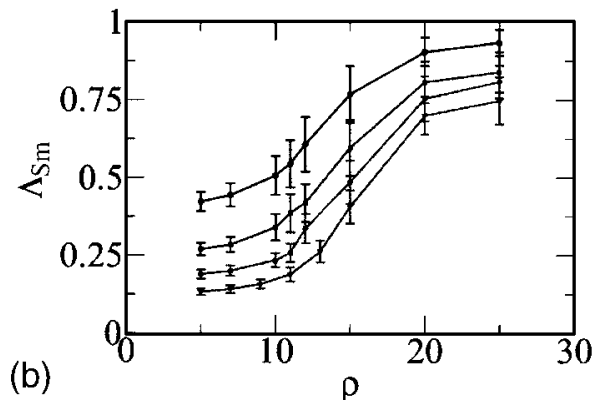

FIG. 8. Dependence of the order parameters with the number of particles $N$ for an arm length of $A=0.3334$. Figure 8(a) shows $S=S(\rho)$ and Fig. 8(b) shows $\Lambda_{\mathrm{sm}}=\Lambda_{\mathrm{sm}}(\rho)$; in both cases $N=100$ (solid circles), 250 (solid squares), 500 (stars) and 1000 (solid triangles) are considered.

$\Phi_{\text {fr }}$ increases faster than $S$ and $\Lambda_{\mathrm{sm}}$. As a general remark, for the extreme values of the arm length, namely, $A \approx 0$ or $A$ $\approx 0.5$, the nematic order parameter $S$ reaches a plateau with its maximum value at smaller densities than the corresponding ones for the smectic order parameter $\Lambda_{\mathrm{sm}}$ and the function $\Phi_{\mathrm{fr}}$. An implication of this observation is that as the density increases, the orientational ordering occurs before the ordering of the positions of the centers of mass of the molecules. This is the reason why the fraction function $\Phi_{\text {fr }}$ remains small for the isotropic and nematic phase. Only for the phases where layers of molecules exist the $\Phi_{\mathrm{fr}}$ function has a steep increment which occurs for low densities.

\section{Nematic phase}

Finally, to discuss the nematic phase a system with molecular arm length $A=0.46$ is chosen. Results for this case are shown in Fig. 6. As before, Fig. 6(a) shows a snapshot of the system, and Fig. 6(b) has the information about the parallel and perpendicular distribution functions which are both randomlike as in the isotropic fluid. This is to be expected since the only characteristic of the nematic phase is the nematic director. Since there is no spatial order of center of mass positions and orientational properties are not reflected in $g_{\|}$ and $g_{\perp}$, they resemble the same features as the isotropic phase. In Fig. 7(d) the nematic and smectic order parameters and the fraction function $\Phi_{\text {fr }}$ are plotted versus the density $\rho$, where it can be seen that the nematic order parameter $S$ increases relatively fast compared to the smectic order parameter $\Lambda_{\mathrm{sm}}$ and the fraction function $\Phi_{\mathrm{fr}}$ which only shows a moderate increase.

\section{E. System size dependence}

To investigate the dependence of the parameters as a function of the system size, simulations were performed for systems consisting of $N=100,250,500$, and 1000 molecules having an arm length of $A=0.3334$. In Fig. 8(a) the nematic order parameter $S(\rho)$ is shown from where it is seen that all curves tend to unity for densities $\rho>20$, independent of the system size. However, for this particular arm length, this fact should not be taken as an evidence for the existence of a nematic phase but as a consequence of the orientational order in the smectic phase. This consideration can be confirmed by the phase map shown in Fig. 2. A different situation occurs with the smectic order parameter $\Lambda_{\mathrm{sm}}$ [see Fig. 8(b)], where there is a noticeable difference for varying numbers of particles, $N$. It can be seen that as the system gets larger, the numerical value of $\Lambda_{\mathrm{sm}}$ decreases. This can be taken as an evidence of missing long range order in the thermodynamic limit.

The reason for the decrement of the smectic order parameter is possibly the appearance of defects, i.e., smectic layers are not infinitely long (in a periodic system), but layers may break while others combine or recombine, resulting in finite length layers located in interstitial positions between smectic layers. This gives rise to nonideal layers and to an induced curvature which results in the observed reduction of the periodicity length $\lambda_{\mathrm{sm}}$. For very big systems, it is expected that not only the smectic but also the nematic order parameter will decrease due to a loss of orientational correlations on large length scales. However, as it is found in the present work, the nematic ordering is more robust with respect to defects. Large scale simulations for the study of long range disorder are in preparation.

\section{F. Constant pressure Monte Carlo}

In order to test the existence of several regions, particularly the defective smectic region, a series of constant pressure Monte Carlo simulations was performed. For the simulations in the isobaric-isothermal ensemble, we performed trial steps where a random walk in $\ln V$ is made. These volume (area) changes were applied to the system by considering a change in only one, randomly selected, dimension of the box at each trial, with a corresponding rescaling of the centers of mass of the particles. This allows the simulation box shape to depart from a perfect square. The acceptance criterion is then evaluated according to the $N p T$-Metropolis algorithm. ${ }^{19}$ The maximum $\ln V$ change was adjusted along the simulation so that approximately $30 \%$ of the volume changes was accepted. In order to get proper statistics several millions of Monte Carlo steps were performed for each pressure.

As a main result the transition from isotropic and smectic phases was found to be in the same place in both ensembles, namely, at the high density border of the defective smectic region. The existence of a defective smectic region was therefore confirmed. It shares characteristics of both the isotropic and smectic phases. More specifically, when the density is increased, the anisotropy starts to develop in a continuous manner. If the density increases from low $\rho \approx 5$ 
TABLE II. Constant pressure and constant volume results for a system of $N=1000$ particles. The density, nematic and smectic order parameters are shown with corresponding standard deviations in both ensembles, $N V T$ and $N p T$. For the defective smectic region, the equilibrated system had very slow relaxation so that the averages had to be taken over $10^{9}$ Monte Carlo steps.

\begin{tabular}{ccccc}
\hline \hline$\rho$ & $P$ & $S$ & $\Lambda_{\mathrm{sm}}$ & Ensemble \\
\hline 5.0 & - & $0.04 \pm 0.02$ & $0.13 \pm 0.01$ & $N V T$ \\
$5.1 \pm 0.06$ & 15 & $0.04 \pm 0.02$ & $0.15 \pm 0.01$ & $N p T$ \\
7.0 & - & $0.06 \pm 0.03$ & $0.14 \pm 0.01$ & $N V T$ \\
$7.21 \pm 0.10$ & 25 & $0.06 \pm 0.03$ & $0.15 \pm 0.01$ & $N p T$ \\
9.0 & - & $0.07 \pm 0.03$ & $0.15 \pm 0.01$ & $N V T$ \\
$9.68 \pm 0.15$ & 35 & $0.10 \pm 0.06$ & $0.19 \pm 0.02$ & $N p T$ \\
11.0 & - & $0.14 \pm 0.07$ & $0.18 \pm 0.02$ & $N V T$ \\
$11.1 \pm 0.23$ & 40 & $0.13 \pm 0.06$ & $0.21 \pm 0.02$ & $N p T$ \\
$13.3 \pm 0.15$ & 45 & $0.47 \pm 0.10$ & $0.30 \pm 0.04$ & $N p T$ \\
15.0 & - & $0.86 \pm 0.02$ & $0.29 \pm 0.04$ & $N V T$ \\
$16.02 \pm 0.34$ & 50 & $0.88 \pm 0.01$ & $0.41 \pm 0.06$ & $N p T$ \\
17.0 & - & $0.93 \pm 0.01$ & $0.40 \pm 0.07$ & $N V T$ \\
$17.4 \pm 0.4$ & 52 & $0.93 \pm 0.01$ & $0.44 \pm 0.05$ & $N p T$ \\
20.0 & - & $0.96 \pm 0.01$ & $0.53 \pm 0.08$ & $N V T$ \\
\hline \hline
\end{tabular}

(typical for the isotropic phase), to $\rho \approx 9$ the molecules start to assemble in order to generate curved layers for which it is not possible to define a smectic director and giving as a net result a regime which is basically isotropic. As the density is increased further, a preferred direction in the system starts to emerge that is defined by a slight majority of layers of molecules. This effect is more evident as the density increases even more, until all layers of molecules are aligned and parallel to each other, defining more clearly a specific direction that corresponds to the smectic director. It may be mentioned that the box lengths $L_{x}$ and $L_{y}$ were found to be rather similar for most of the densities.

In Table II the consistency between the $N V T$ and $N p T$ Monte Carlo simulations results is shown. The nematic and smectic order parameters are shown as a function of density $\rho$, from where it is seen that the order parameters increase continuously as a function of density irrespectively from $N p T$ or NVT. The smectic order parameter is very similar in both ensembles, thereby confirming that the transition to the smectic phase occurs in the same density range in both ensembles. On the other hand, a significant discrepancy is found in the defective smectic region in the values of the nematic order parameter. An examination of the snapshots, however, shows no appreciable nematic order in either case.

\section{SUMMARY}

Extensive Monte Carlo simulations of bent hard needle systems were performed in the NVT ensemble, providing a map of liquid crystal phases. A rich phase behavior was found due to the geometric properties of the model, particularly the existence of the arm length $A$ of the needles. For low enough densities, an isotropic behavior was found for all values of $A$, while for very high densities, the system exhibits a smectic phase for all $A$, except $A=0$ and $A=0.5$ corresponding to the hard needles of Frenkel and Eppenga. ${ }^{2}$ For intermediate values of density $\rho$, the phase map is rather symmetrically centered around $A=0.25$. For small and large
$A$ a nematic phase can be observed. This is to be expected, since molecules behave close to straight needles, for which the nematic phase is well known. ${ }^{2}$ In the central part of the phase map, $A \approx 0.25$, a nontrivial phase behavior of needles was observed. In this region an apparently smooth transition from the isotropic to the smectic phase takes place by the formation of slightly curved clusters, which have no common director. Therefore, short range correlations can be observed in both $g_{\|}(r)$ and $g_{\perp}(r)$. The observed phase transitions may fall in the category of Kosterlitz-Thouless transitions since the intermolecular potential is nonseparable. ${ }^{2}$ In the present paper this type of phase transition is not proven. However, the study of the system size dependence of the smectic order parameter $\Lambda_{\text {sm }}$ for molecular arm length $A=0.3334$ [cf. Fig. 8(b)] may be considered as strong indication for a lack of long range order [increasing the system size tends to decrease the value of $\left.\Lambda_{\mathrm{sm}}(\rho)\right]$.

In order to perform a more robust test for the existence of different phases, in particular, the defective smectic region $N p T$ simulations were performed for the arm length $A$ $=0.18$. The major conclusions from these simulations were the confirmation of the presence of the defective smectic region, and that the transition from the isotropic phase to the smectic one was gradual.

In the present paper only needles with arms orthogonal to the body, e.g., $\theta=\pi / 2$, were investigated. Preliminary results show that in the case of $\theta<\pi / 2,{ }^{21}$ the central region in the phase map, what was called defective smectic, shrinks, i.e., it is observed in a smaller range of arm lengths and for a smaller range of densities. This may be understood from the fact that molecules get closer to the shape of straight hard needles for which no smectic phase is known. ${ }^{2}$ Therefore, also the nontrivial phase of ordered clusters will be less pronounced and will finally vanish for $\theta \rightarrow 0$.

\section{ACKNOWLEDGMENTS}

The authors of this paper acknowledge financial support from Grant No. DGAPA IN-118503, Grant No. DGAPA IN107606, and FENOMEC CPU time. One of the authors (G.S.) gratefully acknowledges financial support from FENOMEC for his stay at UNAM.

\section{APPENDIX A: CALCULATION OF NEMATIC AND SMECTIC ORDER PARAMETERS}

In order to calculate the nematic order parameter, Eq. (2), and the smectic order parameter, Eq. (3), one has to have a reliable estimate for the nematic director $\mathbf{n}$ and both the smectic director $\mathbf{s}$ and the periodicity length $\lambda_{\mathrm{sm}}$ of smectic layers.

The nematic director is straight forwardly calculated as the direction of average orientation of molecules, relative to a given reference frame. The orientational angle is thereby given as 


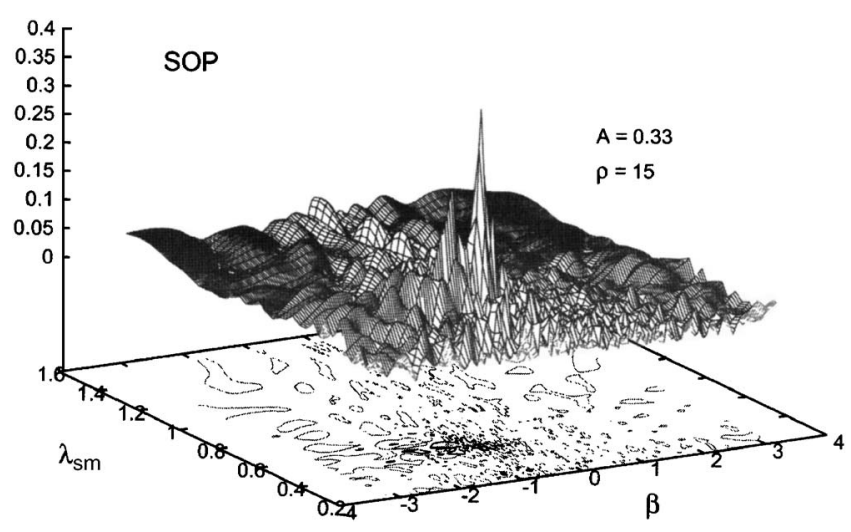

FIG. 9. Surface plot of the smectic order parameter, $\Lambda_{\mathrm{sm}}$, as function of the orientation angle, $\beta$, of the smectic director and the smectic periodicity length $\lambda_{\mathrm{sm}}$. For structures with moderately large order parameters (here $\Lambda_{\mathrm{sm}} \approx 0.4$ ) the parameter landscape is dominated by a large peak around the true maximum position.

$$
\langle\vartheta\rangle=\frac{1}{N} \sum_{i=1}^{N} \vartheta_{i}
$$

where $N$ is the number of bent hard needles in the system and the nematic director $\mathbf{n}$ can be calculated as

$$
\mathbf{n}=(\cos \langle\vartheta\rangle, \sin \langle\vartheta\rangle) .
$$

The calculation of the smectic order parameter is more elaborated. Since molecules may have a different orientation than the smectic director (e.g., smectic $C$ phase), s cannot be calculated as a simple average over molecular orientations. Also, the periodicity length, $\lambda_{\text {sm }}$, cannot be estimated from simple configurational properties. Therefore a possible (but expensive) route to calculate the smectic order parameter would be to make trial estimates for both $\mathbf{s}$ and $\Lambda_{\mathrm{sm}}$ and take those parameters into account for which the smectic order parameter gets its maximum,

$$
\Lambda_{\mathrm{sm}}=\max \left\{S_{\mathrm{sm}}^{\text {trial }}\left(\mathbf{s}, \lambda_{\mathrm{sm}}\right)\right\} \text {. }
$$

In a systematic way all possible parameter combinations in the $\left(\beta, \lambda_{\mathrm{sm}}\right)$ plane are scanned where $\beta$ defines the direction perpendicular to the smectic director $\mathbf{s}$ and is given by Eq. (A5). The resolution of the optimization procedure is thereby given by $\delta \beta$ and $\delta \lambda_{\mathrm{sm}}$, which typically lie in the range of $\pi / 100$ and $1 / 100$, respectively.

This procedure implies a two dimensional optimization procedure, which is rather CPU time consuming when scanning a dense set of possible parameters for $\left(\mathbf{s}, \lambda_{\mathrm{sm}}\right)$.

Therefore an alternative faster scheme is proposed. Fully developed smectic phases are found only for high density systems of bent hard needles and the centers of mass of adjacent particles are significantly close to each other. The proposed procedure consists in finding adjacent particle pairs and calculating the average orientation, $\langle\beta\rangle$, of the distance vector

$$
\langle\beta\rangle=\sum_{i=1}^{N} \arctan \left(\frac{y_{i}-y_{j}}{x_{i}-x_{j}}\right),
$$

where $\quad \mathbf{r}_{j}=\min \left\{\mathbf{r}=\left\{\mathbf{r}_{1}, \ldots, \mathbf{r}_{N}\right\} ;\left|\mathbf{r}_{i}-\mathbf{r}_{j}\right|>d_{\min }\right\} \quad$ and $\quad d_{\min }$ $=\min (A, B)\left(d_{\min }\right.$ is introduced in order to average out very

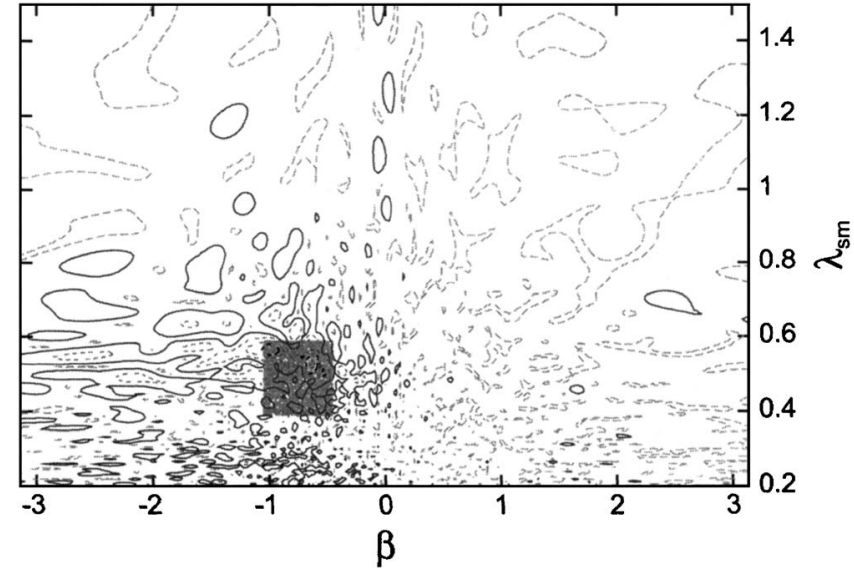

FIG. 10. Contour plot of the same parameter landscape as in Fig. 9. The shaded area indicates the region, in which a refined search of the true maximum position is done. The initial guess, based on the approximate order calculation, as described in the text, is located in the center of this region.

large slopes, which can arise when distances between particles get very small and center of mass coordinates are shifted along their $B$ axis). Note that in a perfect smectic phase, the centers of masses of molecules belonging to one same layer lie on a single straight line with orientation $\beta$ in the Cartesian reference frame. The direction perpendicular to this line is the smectic director. For the nonideal case, the average orientation of the layer is considered and the smectic director is given by

$$
\mathbf{s}=(-\sin \langle\beta\rangle, \cos \langle\beta\rangle)
$$

We note that this nearest neighbor search can be done in $\mathcal{O}(N)$ complexity by using linked-cell lists ${ }^{19,20}$ with cell sizes of $l_{\text {cell }}=1$ (length of one molecule).

The next step consists in calculating $\lambda_{\mathrm{sm}}$. This is done by projecting all particle positions onto the smectic director and building a histogram which gives a distribution of particles along $\mathbf{s}$. Assuming an ideal smectic phase, this would lead to ideal line maxima separated by $\lambda_{\mathrm{sm}}$. In the nonideal case one gets distributions which nevertheless have their maxima

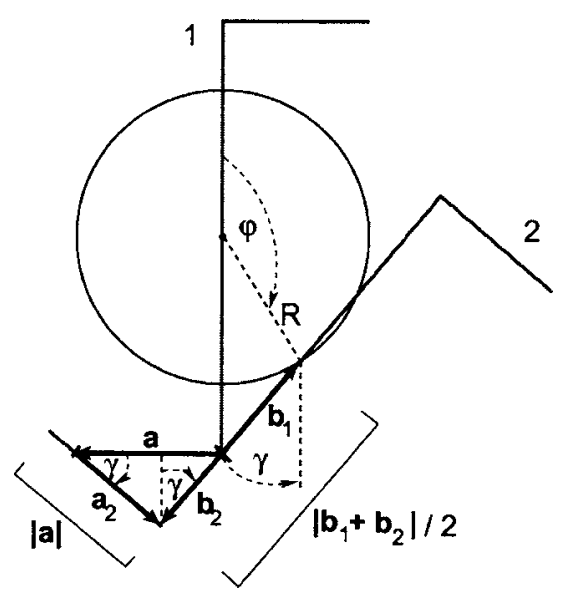

FIG. 11. Schematic view of the set of two molecules forming a maximum angle with each other and fulfilling the minimum distance criterion. 
separated by $\lambda_{\mathrm{sm}}$. The calculation of $\lambda_{\mathrm{sm}}$ is therefore reduced to a simple maximum search within a one dimensional histogram.

It is clear that this method does not provide uniquely the global maximum of the smectic order parameter. However, it gives a reliable estimate for the region in the $\left(\beta, \lambda_{\mathrm{sm}}\right)$ map, where the global maximum is likely to be found. Therefore, the above mentioned method can profitably be used as a preconditioner for a systematic parameter screening, analogous to Eq. (A3). Using the same parameter resolution, $\delta \beta$ and $\delta \lambda_{\mathrm{sm}}$, as for the full scanning procedure, the proposed method is approximately a factor of 100 faster, since the area, which is scanned explicitly corresponds to about $1 \%$ of the full $\left(\beta, \lambda_{\text {sm }}\right)$ plane.

As an example, consider the case for the arm length $A$ $=0.3334$ and density $\rho=15$. Fig. 9 shows $\Lambda_{\text {sm }}$ as function of $\beta$ and $\lambda_{\text {sm }}$, which were scanned with $\delta \beta=\pi / 200$ and $\delta \lambda_{\text {sm }}$ $=1 / 200$. As is found, there is a sharp maximum, corresponding to the true smectic order parameter of the system. Correspondingly, Fig. 10 shows the same data as a contour plot. In this figure, a shaded area is indicated, in which the true maximum is located. It is the same region in which the procedure, outlined above, finds an approximate value for $\Lambda_{\mathrm{sm}}$, which is then refined by scanning the shaded region with the same $\delta \lambda_{\text {sm }}$ and $\delta \beta$, leading to an improved value of $\Lambda_{\mathrm{sm}}$.

\section{APPENDIX B: MAXIMUM ANGLE OF NEAREST NEIGHBOR CRITERION}

In order to calculate the maximum angle $\varphi$ which two molecules may have with each other without overlap and which fulfill at the same time the minimum distance criterion,

$$
d=\sqrt{\left(x_{i}-x_{j}\right)^{2}+\left(y_{i}-y_{j}\right)^{2}} \leqslant R=\min \{A, B / 2\},
$$

a coordinate frame is introduced, where the origin is located at the bottom corner of the first molecule (for a sketch, see Fig. 11). It is anticipated that the maximum angle will be obtained for molecules (i) being a distance $R$ apart and (ii) touching each other in the origin and at the end point of the first molecule. follows:

Therefore the following quantities may be calculated as

(1) Vector, a, along the lower arm of first molecule,

$$
a_{x}=-A, \quad a_{y}=0 .
$$

(2) Center of mass vector, $\mathbf{b}_{1}$, of second molecule,

$$
\begin{aligned}
b_{1 x} & =R \sin \varphi \\
& =\left|\mathbf{b}_{1}\right| \sin \gamma, \\
b_{1 y} & =B / 2+R \cos \varphi \\
& =\left|\mathbf{b}_{1}\right| \cos \gamma .
\end{aligned}
$$

(3) Distance of second molecule from the origin,

$$
b_{1}=\left|\mathbf{b}_{1}\right|=\sqrt{R^{2}+B^{2} / 4+B R \cos \varphi} \text {. }
$$

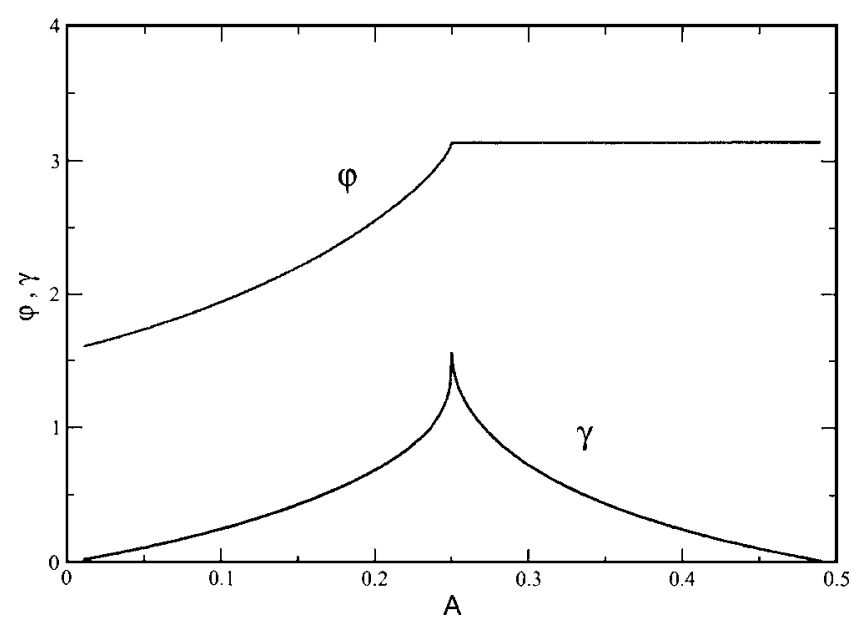

FIG. 12. Maximum angle $\gamma$ between two molecules with arm length $A$ and corresponding angle $\varphi$, obeying the minimum distance criterion.

(4) Vector, $\mathbf{b}_{2}$, pointing to the end of second molecule's body,

$$
\begin{aligned}
b_{2 x} & =-\left(\frac{B}{2}-b_{1}\right) \sin \gamma \\
& =-\frac{B}{2}\left(1-\sqrt{1+4 \frac{R^{2}}{B^{2}}+4 \frac{R}{B} \cos \varphi}\right) \sin \gamma, \\
b_{2 y} & =-\frac{B}{2}\left(1-\sqrt{1+4 \frac{R^{2}}{B^{2}}+4 \frac{R}{B} \cos \varphi}\right) \cos \gamma .
\end{aligned}
$$

(5) Vector, $\mathbf{a}_{2}$, pointing along the lower arm of second molecule,

$$
\begin{aligned}
a_{2 x} & =\frac{b_{2 y}}{\tan \gamma} \\
& =\frac{B}{2}\left(1-\sqrt{\left.1+4 \frac{R^{2}}{B^{2}}+4 \frac{R}{B} \cos \varphi\right) \frac{\cos ^{2} \gamma}{\sin \gamma},}\right. \\
a_{2 y} & =b_{2 y} .
\end{aligned}
$$

(6) Angle of orientation $\gamma$, of second molecule with respect to body of first molecule [follows from Eqs. (B3) and (B4)],

$$
\gamma=\sin ^{-1}\left\{\frac{2 R \sin \varphi}{B \sqrt{1+4\left(R^{2} / B^{2}\right)+4(R / B) \cos \varphi}}\right\} .
$$

Now one can formulate the problem as follows: find the angle $\varphi$ for which both molecules are in touch with each other (i) at the origin and (ii) the end of the lower arm of the first molecule, i.e.,

$$
\mathbf{a}+\mathbf{a}_{2}=\mathbf{b}_{2}
$$

or

$$
a_{x}+a_{2 x}-b_{2 x}=0 \text {, }
$$




$$
a_{y}+a_{2 y}-b_{2 y}=0 \text {. }
$$

Because of Eqs. (B2) and (B13) the condition Eq. (B17) is trivially fulfilled, so that the only task is to solve Eq. (B16), which is written explicitly as

$$
A-\left(1-\sqrt{1+4 \frac{R^{2}}{B^{2}}+4 \frac{R}{B} \cos \varphi}\right) \frac{1}{\sin \gamma(\varphi)}=0 .
$$

Here the dependence of $\gamma$ of the angle $\varphi$ was explicitly noted. Therefore, knowing $\varphi$, the angle $\gamma$ is properly calculated. Unfortunately, this problem has no analytical solution due the complicated dependence on the angle $\varphi$. However, it may be obtained numerically.

Figure 12 shows the behavior of $\gamma_{\max }$ as well as the optimum angle $\varphi$. For smallest arms, $\varphi \approx \pi / 2$, which means a parallel orientation. This shows that those molecules are unlikely to form clusters of curved shape. The excluded volume of these molecules is so small that very close molecules can orient only parallel to each other. In dense phase, molecules for apart will be oriented with a similar director, which is the nematic phase. But the smectic phase is only obtained for very high densities and $A>0$, which may be understood as a packing effect. For $A=0$ there is no such packing effect and the smectic phase is absent. ${ }^{2}$ For larger values of the arm the maximum angle $\varphi$ tends to $\pi$. This means that those molecules are very likely to form local clusters of curved shape as it is observed in the phase map (see Fig. 2). These local clusters can then be understood as condensation nuclei for a smectic phase. The angle $\gamma$ shows a nontrivial dependence on $\varphi$ and $A$. It has a sharp maximum at $A=0.25$, where $\gamma=\pi / 2$. This result comes out from the fact that $A=B / 2$ in this special case. For larger arms $\gamma$ decreases again taking values $\gamma \rightarrow \pi / 2$ for values of $A \rightarrow 1 / 2$. The $\gamma$ dependence of the arm length $A$ is nearly symmetrically centered around $A=0.25$. This is a strong indicator of two corresponding arm lengths $A_{1}=0.25-x$ and $A_{2}=0.25+x$ $(x \in[0,0.25])$, having equivalent geometric properties. This would also explain the rather symmetrical distribution of phases in the observed phase map.

${ }^{1}$ R. A. Perusquia, J. Peon, and J. Quintana, Physica A 345, 130 (2005).

${ }^{2}$ D. Frenkel and R. Eppenga, Phys. Rev. A 31, 1776 (1985).

${ }^{3}$ Multipolar interactions have been added, for example, M. Houssa, L. F. Rull, and S. C. McGrother, J. Chem. Phys. 109, 9529 (1998); R. Berardi, S. Orlandi, and C. Zannoni, Chem. Phys. Lett. 261, 357 (1996); S. J. Johnston, R. J. Low, and M. P. Neal, Phys. Rev. E 66, 061702 (2002).

${ }^{4}$ A. Cuetos, B. Martinez-Haya, S. Lago, and L. F. Rull, Phys. Rev. E 68, 011704 (2003).

${ }^{5}$ V. Palermo, F. Biscarini, and C. Zannoni, Phys. Rev. E 57, 2519 (1998).

${ }^{6}$ R. Berardi, H.-G. Kuball, R. Memmer, and C. Zannoni, J. Chem. Soc., Faraday Trans. 94, 1229 (1998).

${ }^{7}$ R. Berardi, M. Ricci, and C. Zannoni, ChemPhysChem 2, 443 (2001).

${ }^{8}$ C. Zannoni, J. Mater. Chem. 11, 2637 (2001); R. A. Bemrose, C. M. Care, D. J. Cleaver, and M. P. Neal, Mol. Phys. 90, 625 (1997).

${ }^{9}$ D. Frenkel and B. M. Mulder, Mol. Phys. 55, 1171 (1985).

${ }^{10}$ D. Frenkel, H. N. W. Lekkerkerker, and A. Stroobants, Nature (London) 332, 822 (1988).

${ }^{11}$ L. Onsager, Ann. N.Y. Acad. Sci. 51, 627 (1949).

${ }^{12}$ A. G. Vanakaras, D. Photinos, and E. T. Samulski, Phys. Rev. E 57, R4875 (1998).

${ }^{13}$ J. M. Polson and D. Frenkel, Phys. Rev. E 56, R6260 (1997).

${ }^{14}$ J. Saucedo-Zugazagoitia, B.S. Thesis, Universidad Nacional Autonoma de México, 2006.

${ }^{15}$ F. Pucheta-Mendez, B.S. Thesis, Universidad Nacional Autonoma de Mexico, 2005

${ }^{16}$ This kind of plot is necessary for those cases where the density is high and it is very difficult to see the structure of the configuration, for this reason from now on most of the snapshots presented in this paper, are drawn following this criterion.

${ }^{17}$ For those cases where there is not an anisotropic behavior, the parallel and perpendicular directions are arbitrary.

${ }^{18}$ It is convenient to note that a large value of $S$ does not necessarily imply a nematic phase, as in this case. This is only a coincidence that in the smectic phase orientational order also exists.

${ }^{19}$ D. Frenkel and B. Smit, Understanding Molecular Simulation (Academic, San Diego, 1996).

${ }^{20}$ G. Sutmann and V. Stegailov, J. Mol. Liq. 125, 197 (2006)

${ }^{21}$ R. A. Perusquia, M. Sc. thesis, Universidad Nacional Autonoma de México, 2004 